\title{
EL APRENDIZAJE BASADO EN PROBLEMAS (ABP) EN LA EDUCACIÓN MATEMÁTICA EN COLOMBIA. AVANCES DE UNA REVISIÓN DOCUMENTAL.
} PROBLEM- BASED LEARNING (PBL) IN MATHEMATICS EDUCATION IN COLOMBIA. ADVANCES OF A DOCUMENTARY REVIEW

\section{Padilla-Doria Luis Alfonso' ${ }^{1}$}

Flórez-Nisperuza Elvira Patricia²

\section{RESUMEN}

El aprendizaje basado en problemas (ABP) ha resultado ser una estrategia didáctica implementada a nivel mundial en distintas modalidades escolares y niveles. En este estudio, se revisan estudios llevados a cabo a nivel internacional, nacional y local en relación al uso de esta estrategia en la educación matemática y en particular, en la formación de profesores de esta área del currículo escolar colombiano. Los resultados indican que el ABP mejora las prácticas de enseñanza y aprendizaje de las matemáticas de manera consistente y 1 Docente I.E. Germán Vargas Cantillo. Cereté, Colombia. sekama2013@gmail.com Código ORCID: 00000002-5431-7324. : Doctorando de Universidad UMECIT, Panamá. luispadilla.est@umecit.edu.pa

$2 \quad P h D$. Docente titular Universidad de Córdoba. Montería, Colombia. epatriciaflorez@correo.unicordoba. edu.co. https://orcid.org/0000-0003-4621-8382 que en base a la evidencia debería promoverse más su uso.

PALABRAS CLAVE: Aprendizaje basado en problemas, educación matemática, estrategia didáctica.

\section{ABSTRACT}

Problem-based learning (PBL) has proved to be a useful and consistent teaching strategy, to the point that it has begun to be implemented worldwide in different school modalities and levels. In this study, we will review the studies carried out at international, national and local levels in relation to the use of this strategy in mathematics education and in particular in the training of teachers in this area of the Colombian school curriculum. The results indicate that PBL 
consistently improves mathematics teaching and learning practices and that, based on the evidence, its use should be further promoted.

KEY WORDS: Problem-based learning, mathematics education, didactic strategy

\section{INTRODUCCIÓN}

El aprendizaje basado en problemas (ABP) se ha perfilado como una estrategia didáctica transversal, en el sentido no solo de que puede ser usado en distintas disciplinas, sino que también facilita el trabajo interdisciplinario entre ellas. Puntualmente, en el área de la enseñanza de las matemáticas, ha resultado especialmente útil en cuanto ha permitido poner en contexto a las matemáticas, desafío no siempre sencillo o factible.

En este marco, tanto la formación del docente de matemáticas, así como sus prácticas en distintos niveles educativos, se han visto atravesadas por muchos esfuerzos e investigaciones con relación a las posibilidades que ha dado el ABP. En este artículo se realiza una revisión documental y sistematización del $\mathrm{ABP}$ en los últimos cinco años, con relación a la enseñanza de las matemáticas, abarcando un marco internacional hasta los casos llevados en territorio colombiano.

\section{DESARROLLO}

La formación del docente de matemáticas es un tema ampliamente abordado que comprende niveles para su descripción (Linares, 2018), van desde los aspectos vinculados a la enseñanza de contenidos, hasta discusiones sobre la formación docente en distintos niveles. A lo largo de la revisión, se concluye que el proceso de formación docente en general, incluyendo a la educación en matemáticas, constituye un proceso contextualizado y multifacético, con variadas características y énfasis de acuerdo con cada lugar y conjunto actores.
Un primer grupo de investigaciones se refiere al campo de la enseñanza de las matemáticas en los distintos escenarios en los últimos años. Así, el estudio de Gutiérrez (2017) en Estados Unidos llamado Political knowledge for teaching mathematics, precisa que para una buena enseñanza de las matemáticas es suficiente un conocimiento profundo y comprensivo por parte del docente de los conocimientos a enseñar; o el desarrollo de un buen vínculo con el estudiantado. Por ello, muestra interés en los factores políticos que suelen ser causa de las dificultades de enseñanza por parte de los docentes en el marco de las grandes discusiones y cambios requeridos en el currículo escolar y señala que dichos factores deberían ser analizados en cualquier otro contexto educativo, entendiendo que toda enseñanza es política en alguna medida.

Por su parte, el estudio realizado en la India por Muhtadi, Kartasasmita y Prahmana en el mismo año, indaga acerca de la eficacia de la incorporación de software (TPACK) para la enseñanza de matemática específico, revelando una mejora general de la comprensión de los temas, así como el rendimiento de los estudiantes, llegando a la conclusión de que más escuelas deberían estimular el desarrollo de programas y sistemas construidos con base a sus propias necesidades pedagógicas.

Asimismo, Dele-Ajayi et al. (2019) en Nigeria optan por un enfoque similar en un estudio teórico que plantea una fuerte crítica al tradicionalismo que aun tiende a regir en cuanto a la dinámica de la enseñanza de las matemáticas. Indican que la realidad de los estudiantes de los distintos niveles educativos ha cambiado radicalmente en las últimas décadas, pero la manera de enseñar pareciera que hace caso omiso a esto, manteniendo prácticas que resultan contraproducentes. 
En este orden, se registra la investigación de Thurm y Barzel (2020) en Alemania que revela la existencia de una correlación significativa entre las actitudes y creencias positivas hacia la tecnología en general, su incorporación en la práctica diaria (esté o no reglamentada o por escrito en las planificaciones) y la eficacia e interés por parte del estudiantado en la clase de matemática.

En el continente europeo, se destaca el artículo de revisión de Barakaev et al. (2020) que aborda las problemáticas de las últimas décadas relacionadas con la enseñanza de la matemática en América y Europa, señalando como ésta es cada vez más necesitada en muchas áreas de especialidad, pese a que cada vez se presentan más dificultades para su abordaje y buscan por tanto delimitar el rol de la didáctica de la matemática como un rol estrictamente pedagógico antes que técnico.

En el mismo ámbito, pero desde un marco de estudio experimental mediante grupo de estudio y grupo control, se destaca la tesis doctoral de la peruana Neyra-Quezada (2020) que indaga el efecto del empleo del binomio aprendizaje significativo en conjunción con el aprendizaje basado en problemas, encontrando que hay diferencias significativas a favor del uso de aprendizaje basado en problemas en cuanto a la enseñanza de las matemáticas.

De igual manera, surgen grupos de trabajos específicos en áreas de interés reciente orientados a entender el lugar de la tecnología en la enseñanza de las matemáticas y cómo en sus distintas opciones se promueven habilidades y aprendizajes. Así, Uzbekistan, Rashidov (2020) estudia el rendimiento de grupos diferenciados entre estudiantes que, si bien tenían todo el mismo nivel de conocimiento matemático, presentaban diferencias en cuanto al acceso a la tecnología lo que produjo calificaciones que incorporaban tareas mediadas por tecnología en el aula. Esto, que parece algo evidente, tiene como consecuencia reconocer que la alfabetización digital es determinante en el desarrollo de la enseñanza de la matemática.

Igualmente, el estudio de Demitriadou, Stavroulia y Lanitis (2020) en Grecia incorpora el uso y evaluación de realidad aumentada para la enseñanza de matemáticas en el nivel primario en el marco directo del aprendizaje basado en problemas, mostrando resultados tanto en términos del interés generado en todo el estudiantado, así como en su capacidad para resolver problemas matemáticos derivados de esta experiencia de realidad aumentada.

Finalmente, en un estudio realizado en Rusia en el presente año, Soboleva et al. señalan los beneficios de la incorporación del pensamiento computacional en ejercicios de matemática a través del uso de videojuegos para estimular el aprendizaje de matemática, despertando no solo el interés de los estudiantes sino también promoviendo de manera eficaz capacidades de lógica, cálculo y resolución de problemas en toma de decisiones. Hay que destacar que todos estos videojuegos se relacionan con ejercicios matemáticos construidos sobre problemáticas concretas, empleando el enfoque de aprendizaje basado en problemas.

Un segundo campo temático revisado, se refiere a las relaciones de la epistemología con los conocimientos teóricos en la enseñanza de la matemática. El trabajo de Maher et al. (2018) en los Estados Unidos concluye que los primeros acercamientos didácticos que los estudiantes tienen con la matemática (generalmente vinculados más a la forma de ser del docente y sus actitudes antes que el contenido en sí), modelan en la actitud posterior y sostenida hacia esta disciplina en el resto de la trayectoria educativa. Es decir, tal como destacan otros estudios similares (Baky y Gursöy, 2018; Deringol, 2018, ambos en Turquía) las ideas que los alumnos desarrollan hacia los contenidos matemáticos son predictores del rendimiento 
futuro, en especial cuando esto genera fuerte ansiedad e incertidumbre a muy temprana edad.

En tercer lugar, en lo que respecta a enseñanza de las matemáticas y sus relaciones con el ABP, se encuentra la investigación Simamora, Sidabutar y Surya realizada en Indonesia (2017) que comprueba la capacidad de resolución de problemas matemáticos en el nivel primario, hallando que el grupo de niños cuyos docentes aplicaron $\mathrm{ABP}$ resolvían significativamente mejor los problemas matemáticos que el grupo que no lo aplicó. Resultados similares se hallan en otro estudio llevado también en Indonesia por Firdaus (2017), que integra el uso de la instrucción directa como estrategia complementaria y factor aglomerado de la ABP.

Por su parte, La Force, Noble y Blackwell (2017) en Estados Unidos estudian correlaciones entre la implementación de la ABP y la motivación de estudiantes con relación al aprendizaje de las matemáticas y señalan que en los grupos donde el ABP era implementado como estrategia didáctica, la predisposición hacia la clase de matemática y la motivación hacia las actividades tanto presenciales como en casa resulta significativamente mayor que en otros grupos.

En este orden, se reporta la tesis doctoral de los Peruanos Godoy y Carter (2020) emplea un diseño experimental. La muestra estuvo conformada por 45 estudiantes distribuidos en dos grupos: 22 estudiantes para el grupo control y 23 estudiantes para el grupo experimental, encontrando resultados similares, a favor de los grupos de estudiantes que trabajaron con ABP.

En concordancia con ello, la tesis doctoral de Soledad (2020) lista y pone a prueba mediante rendimiento de grupos distintas estrategias a partir del aprendizaje basado en problemas para fortalecer las competencias matemáticas en el nivel primario, encontrando que las estrategias basadas $\mathrm{u}$ orientadas hacia aprendizaje basado en problemas dan mejores resultados que las otras.

Las conclusiones y acuerdos con respecto a las bondades del empleo del ABP en distintos países, también tienen su reflejo en el territorio nacional. No obstante, en primera instancia se relevan seguidamente los estudios más recientes con relación a la matemática y su enseñanza, para luego señalar en específico aquellos que emplean el $\mathrm{ABP}$ como uno de sus factores.

En el ámbito colombiano, se relevan principalmente cuatro grandes grupos de estudios. Aquellos vinculados al nivel de conocimiento matemática tanto en estudiantes como docentes, otros vinculados a la relación entre la enseñanza de la matemática y las condiciones materiales, un grupo de estudios propiamente sobre didáctica de la matemática y su epistemología, y los relacionados con cambios generados por el COVID-19.

En el primer grupo, se destaca el estudio de Hernández-Suárez, Prada-Núñez y GamboaSuárez (2017) quienes diagnosticaron el nivel de conocimiento matemático y conocimiento sobre lenguaje matemático en estudiantes de primeros años de profesorados en matemáticas en escuelas del municipio del Norte de Santander, encontrando que no solo el nivel de conocimiento matemática tiende a ser bajo, sino que también hay grandes dificultades con relación al dominio del lenguaje propio de la disciplina. Mendoza, Burbano y Valdivieso en el año 2019 en el departamento de Boyacá, analizaron el conocimiento matemático de un conjunto de docentes de nivel básico que daban matemática en entornos virtuales y encontraron resultados preocupantemente bajos. Lubert, Ruiz-Ortega y Londoño (2021) hallaron que, en este tipo de resultados, era muy importante el nivel de herramientas metacognitivas y su presencia en los docentes que si tenían buenas puntuaciones en el área de enseñanza de su 
propia especialidad, que parecía extenderse incluso a áreas de conocimiento general.

En relación con el segundo grupo de estudios dedicados al tema de las condiciones materiales y recursos a la hora de abordar la enseñanza de las matemáticas, se reporta la investigación de Lezcano-Brito, Mary-Benítez y CuevasMartínez (2017) llevado a cabo en Bogotá, que evalúa y describe las ventajas del uso de las TIC para la enseñanza de las matemáticas. Puntualmente, se trabaja con un programa diseñado y programado para la enseñanza de los números naturales, presentando resultados alentadores. En el departamento del Norte de Santander, Bautista-Rico (2017) propone la incorporación de TIC en enseñanza de la matemática, pero desde una perspectiva de resolución de problemas cotidianos, buscando facilitar ciertas tareas que son repetitivas y no requieren pensamiento matemático pero que están involucradas en la matemática y pueden funcionar como obstáculos. Otro estudio (MatoVázquez, Chao-Fernández, Chao-Fernández, 2019) encontraron resultados positivos en la enseñanza de matemáticas en el nivel primario mediante el empleo de música, a través de investigaciones con grupo experimental y control, con resultados estadísticamente significativos en todas las áreas a favor del grupo que recibió enseñanza a través de actividades musicales.

Otros estudios orientados a investigar y mejorar la planificación y didáctica de las matemáticas desde sus relaciones con la epistemología, revelan investigaciones como la de SánchezLuján en México (2017) o Alsina en Ecuador (2019) donde se repasan metodologías y perspectivas epistemológicas que actúan como base para el desarrollo de propuestas concretas en el nivel de educación básico. Otro estudios, definen modelo pedagógicos como el de Luna y Páez (2018), donde se postula también que lo más importante para la enseñanza de las matemáticas es el énfasis en lo pedagógico antes que en el contenido. Este tipo de enfoque se refuerza en estudios como el de Pérez-González, Valdés-Rojas y GarrigaGonzález (2019) en su investigación llevada a cabo en Cuba, desarrollan una propuesta integral de formación en planificación didáctica, que pueda ser adaptada a distintos contextos de enseñanza, basándose en las fortalezas de las habilidades profesionales propias de los docentes, buscando apoyarse más en las fortalezas del cuerpo docente antes que en sus debilidades. En la misma área, pero desde una perspectiva opuesta, trabajos como el de da Silva y Manrique (2021), retoman el conocimiento de un grupo de profesores de matemática que trabajan en nivel inicial, teniendo ellos formación de base matemática y destacan que es imposible constituir un aprendizaje realmente significativo sin un conocimiento al menos sólido de los contenidos matemáticos a enseñar. Esto pone sobre la mesa uno de los debates que se ha dado no solo en el campo de las matemáticas, sino de las disciplinas en general. ¿Qué es más importante para enseñar, lo pedagógico, o lo disciplinar? Si bien ha habido mucho debate alrededor, cualquier postura sensata señalará que ambos aspectos tienden a ser excluyentes cada uno y se necesitan ambos.

En ámbitos similares, la tesis doctoral de Cadavid (2017) en Colombia rescata las voces y experiencias de maestras del nivel básico que enseñan matemáticas, donde uno de los factores que salen en los resultados es la incomodidad para con el contenido, así como la incomodidad ante la resistencia de algunos alumnos hacia la matemática. Otro artículo reciente que retoma estos asuntos es el de Alsina (2019b) en Ecuador, que señala que nunca ha habido tanta investigación ni interés en la didáctica de las matemáticas como en las últimas dos décadas, pero que muchas veces el cúmulo de conocimientos generado, si bien es valioso y cuantioso, no tiene una transferencia directa para con los responsables de la enseñanza en 
los niveles iniciales y básicos. En relación con lo planteado la propuesta de esta investigación, se reportan estudios como el de Rodríguez (2020), que diagnostica los conocimientos específicos metodológicos vinculados a enseñanza de las matemáticas en cuerpos docentes para constituir planes de capacitación.

En el cuarto grupo, Jiménez (2020) en Costa Rica propone el desarrollo de formación para formadores tomando como eje el análisis de los planes de estudio, para analizar las debilidades en el marco de la pandemia por COVID-19 y reforzarlas en programas de e-learning tanto para docentes como para alumnos. En este tema de la pandemia, se encuentra la investigación llevada a cabo en Cuba de Lezcano, Puy y Puy (2021) que explica los factores que se tuvieron en cuenta a la hora de gestionar un plan para la enseñanza de la matemática en el marco de la virtualidad, donde se destacan dos acciones principales. La primera, el rediseño de los materiales educativos y la constitución de una biblioteca con material de calidad y digital, totalmente accesible. La segunda, la incorporación definitiva de las TIC a los procesos de enseñanza aprendizaje. Este aspecto de las TIC ha sido recalcado en decenas de estudios en pandemia, y se destaca la investigación realizada por Pachas (2020) en Perú lista y documenta recursos TIC para la enseñanza de las matemáticas, poniendo énfasis en aquellos que se volvieron necesarios y casi obligatorios durante la pandemia.

Desde otro enfoque, orientado a la experiencia docente antes que los recursos, se registra el estudio de Pérez-Díaz (2021) en México y el de Izasa, Suárez y Leal (2019) en Colombia, que reflexionan sobre los factores que determinan la posibilidad de desarrollar un acto de enseñanza y aprendizaje por fuera de la tecnología relacionado con tener un espacio adecuado, apoyo familiar del lado del estudiante, dimensiones emocionales, entre otros. En este marco, el docente muchas veces debe convertirse en aquello que falta y llenar huecos o improvisar para poder llevar la clase adelante.

A través de esta revisión, se ha puesto en evidencia la importancia de factores relevantes para mejorar los procesos de enseñanza de las matemáticas y reforzar la formación docente. Entre ellos, enseñar a través de problemas y su contextualización paramejorarsignificativamente la predisposición de los estudiantes hacia el aprendizaje de una disciplina que suele resultar compleja, en especial, en los niveles iniciales.

Con relación a lo expuesto, y ahondando sobre la implementación del $A B P$ en el área de las matemáticas existen numerosas investigaciones y propuestas que dan cuenta de que puede ser un método efectivo a la hora de plantear otra metodología de enseñanza distinta a la tradicional. Según las investigaciones mencionadas en esta oportunidad, el ABP favorece y estimula varias capacidades de los educandos favoreciendo el proceso de enseñanza-aprendizaje. A continuación, se citan las investigaciones encontradas en el ámbito de Colombia en las que se trabaja con ABP y enseñanza de las matemáticas de relación directa y aportes sustanciales a la pertinencia de una propuesta doctoral en curso.

Así, la investigación llevada a cabo en Boyacá por Vargas (2019) denominada "Aprendizaje basado en problemas mediados por TIC para superar dificultades en el aprendizaje de operaciones básicas matemáticas", hace referencia a la importancia de aplicar dicho método, concluyendo que el nivel de aprendizaje de los alumnos mejora su implementación. En los resultados obtenidos en la investigación se logra establecer que el nivel más alto de aprendizaje de los estudiantes se refleja cuando se trabaja a través de la metodología del ABP. (Vargas, 2019). 
Por su parte, la investigación realizada en Córdoba por Rodríguez Tortosa (2018) denominada "ABP una propuesta para la clase de matemáticas", propone la implementación del método a través del trabajo interdisciplinar con otras áreas y enfatiza en la postura de los alumnos a la hora de aprender con dicho método, donde según los resultados el nivel de motivación fue alto y los alumnos estuvieron a gusto con otra forma de aprender distinta al método tradicional. Tras confirmar en primera persona, todos y cada uno de los beneficios que se le atribuyen a esta metodología, me gustaría destacar algo que me sorprendió: la facilidad con la que los alumnos se habituaron al cambio. Era la primera vez que trabajaban con una metodología diferente a la tradicional y desde la primera sesión (tras presentar el proyecto y explicar la dinámica de trabajo) se sintieron ilusionados y motivados con el proyecto. Al finalizar el mismo indicaron en el cuestionario que les gustaría seguir trabajando con esta metodología durante el resto del curso.

A su vez, en la investigación realizada en Boyacá por Cristancho Cárdenas (2018), se propone la aplicación del ABP para la enseñanza de fracciones desarrollando habilidades comunicativas y trabajo en grupo, logrando que los estudiantes sean más competentes en la resolución de problemas y en particular, las capacidades de análisis frente a situaciones problema particulares.

Otra investigación relevante fue la realizada por Matamorros Espinoza (2018) en Curitiba donde se plantea una "Propuesta Didáctica de Aprendizaje basado en Problemas dirigida al área de matemáticas $\left(8^{\circ}\right.$ de educación general básica): Caso Unidad Educativa "Sagrada Familia". En esta, hace referencia a que el ABP es una metodología útil para satisfacer las necesidades pedagógicas contemporáneas y permite a los docentes demostrar el dominio que tienen sobre la materia que imparten, al igual que su capacidad pedagógica; en tanto que para los alumnos se convierte en un mecanismo útil para el desarrollo de sus capacidades y habilidades, como, por ejemplo, el análisis, síntesis, argumentación, interpretación, pensamiento crítico, trabajo en equipo, entre otros.

Además, el estudio de Escobedo et al. (2018) en Veracruz, propone a las matemáticas como conocimiento transversal a través del ABP para la enseñanza de Ciencias de la Computación y Física. A lo largo de un semestre, se propuso la enseñanza de matemáticas de forma transversal con relación directa a las otras disciplinas, y se observó un mejor desempeño en todas las materias en su conjunto.

Vélez et al. (2020), correlacionan el empleo del ABP en matemáticas con la mejora de desempeño en razonamiento lógico y deductivo en alumnos de la educación general básica. Metodológicamente se fundamentó desde una investigación tipo descriptiva correlacional no experimental transversal, para la recolección de información se empleó la encuesta y un cuestionario elaboradas en escala Likert, aplicada en un único momento. Con los resultados obtenidos se confirma el objetivo de la investigación; mismo que, propone la aplicación del ABP como estrategia didáctica para el desarrollo del razonamiento lógico matemático en los estudiantes, motivando y potenciando de una manera efectiva y rápida la resolución de problemas con diferentes alternativas y como nuevo método de aprendizaje de las matemáticas.

Resultados similares se obtienen en el estudio de Vargas-Pulido (2021) que indaga acerca del aprendizaje basado en problemas y su influencia en el aprendizaje de sistema de ecuaciones lineales 2x2 en los Estudiantes del 9-4 de la Institución Educativa Nuestra Señora de los Dolores de Manare de Villanueva Casanare, en Colombia. La metodología utilizada fue variada de acuerdo a los intereses y necesidades de los estudiante buscando siempre que los 
educandos aplicasen sistemas de ecuaciones $2 \times 2$, de acuerdo a ello, se aplicó un pre- test que determinó el nivel de comprensión de las diferentes competencias matemáticas con el ánimo de contrastar el mismo test al final del proceso seguidamente se ejecutó de manera secuencial la estrategia didáctica del aprendizaje basado en problemas enmarcado en una pregunta direccionadora; los resultados que se obtuvieron en la investigación demostraron que el aprendizaje basado en problemas es una estrategia eficaz en el proceso de aprendizaje del sistema de ecuaciones lineales $2 \times 2$, confirmando que su implementación en general conlleva a la mejora de desempeño en contraste con los grupos donde no se aplica.

También se reporta el estudio de Berardi y Corica (2021) en Sevilla, que emplea el ABP como una forma de constituir un aprendizaje interdisciplinario entre las matemáticas y otras disciplinas afines. En este estudio, se desarrollaron proyectos de aprendizaje de manera conjunta entre las materias de Ciencias Naturales y Matemáticas mediante problemas en la clase de Ciencias Naturales, en los cuales las matemáticas cumplían un rol determinante para su resolución. A través de un trabajo de meses continuos, se pudo observar y medir que en efecto el aprendizaje de las matemáticas se vio mucho más consolidado que en los casos en que no se realizó este tipo de trabajo conjunto.

\section{CONCLUSIONES}

A lo largo de esta revisión, se ha puesto de manifiesto que el $\mathrm{ABP}$ es una herramienta que ha sido aplicada en una gran cantidad de campos de la enseñanza de las matemáticas, que van desde aspectos generales como el razonamiento lógico hasta elementos específicos del algebra. Se ha podido observar que, en general, los deiferentes estudios con sus distintos enfoques, muestran resultados prometedores para esta metodología. No obstante, hacen falta estudios orientados al metaanálisis en este campo al menos para la región Caribe colombiana, abriendo el campo y línea de investigación asociada a estudiar el ABP en la educación matemáticas como fundamento para la formación de profesores en esta área del currículo escolar colombiano.

\section{REFERENCIAS BIBLIOGRÁFICAS}

Alsina, À. (2019a). Itinerarios didácticos para la enseñanza de las matemáticas (6-12 años). Números. Revista de Didáctica de las Matemáticas, 101, 215-216.

Alsina, À. (2019b). La educación matemática infantil en España: ¿qué falta por hacer? Números: revista didáctica de las matemáticas, 2019(100), 187-192.

Baki, A., \& Gürsoy, K. (2018). Does using history of mathematics make sense? The views of teacher candidates. Journal of Pedagogical Research, 2(2), 78-90.

Barakaev, M., Shamshiyev, A., O'rinov, X., \& Abduraxmonov, D. (2020). Problems of Teaching Mathematics in Modernization. International Journal of Progressive Sciences and Technologies, 19(2), 201-203

Bautista-Rico, R. Y. (2017). El uso didáctico de las TICS en el mejoramiento de la labor didáctica en la escuela colombiana. Aibi revista de investigación, administración e ingeniería, 5(2), 2-8.

Berardi, E. Á., \& Corica, A. R. (2021). ABP en la escuela secundaria: análisis de la gestión de un proyecto sobre el crecimiento de plantas y sus vínculos con la matemática. Épsilon.

Cadavid, L. A. (2017). Constitución de la subjetividad del sujeto maestro que enseña matemáticas, desde y para la actividad pedagógica [Disertación Doctoral, Universidad de Antioquia]. 
Cristancho Cárdenas, D. (2018). Aprendizaje basado en problemas en matemáticas: el concepto de fracción. Revista Educación y Ciencia, 21, 45-58.

Dele-Ajayi, O., Strachan, R., Pickard, A. J., \& Sanderson, J. J. (2019). Games for teaching mathematics in Nigeria: what happens to pupils' engagement and traditional classroom dynamics? IEEE Access, 7, 53248-53261.

Demitriadou, E., Stavroulia, K. E., \& Lanitis, A. (2020). Comparative evaluation of virtual and augmented reality for teaching mathematics in primary education. Education and Information Technologies, 25(1), 381-401.

Escobedo, J. F. C., Trejo, F., Ortiz, M. R. S., \& Gonzalez, F. M. (2018). Aplicación Didáctica del ABP en Computación Básica, Física y Álgebra en la enseñanza de la Ingeniería en la Universidad Veracruzana Campus Coatzacoalcos. Revista de Docencia e Investigación Educativa, 4(13), 10-20.

Firdaus, F. M. (2017). Improving Primary Students' Mathematical Literacy through Problem Based Learning and Direct Instruction. Educational Research and Reviews, 12(4), 212-219.

Gutiérrez, R. (2017). Political conocimiento for teaching mathematics. Building support for scholarly practices in mathematics methods, 11.

Hernández-Suárez, C. A., Prada-Núñez, R., \& Gamboa-Suárez, A. A. (2017). Conocimiento y uso del lenguaje matemático en la formación inicial de docentes en matemáticas. Revista de investigación, desarrollo e innovación, 7(2), 287-299.
Izasa, W. B. P., Suárez, C. A. H., \& Leal, O. L. R. (2019). Estrategias para la enseñanza de la matemática: una mirada desde los docentes en formación. Revista Perspectivas, 4(1), 48-53.

Jiménez, Y. R. (2020). Propuesta para enseñar y aprender matemática a distancia desde un abordaje novedoso de los Programas de Estudio. Cuadernos de Investigación y Formación en Educación Matemática, (19), 147-163.

LaForce, M., Noble, E., \& Blackwell, C. (2017). Problem-based learning (PBL) and student interest in STEM careers: The roles of motivation and ability beliefs. Education Sciences, 7(4), 92.

Lezcano, A., Puy, J., \& Puy, A. (2021). De la presencialidad a la virtualidad: Enseñar Matemáticas en Pandemia. TE \& ET.

Lezcano Brito, M., Mary Benítez, L., \& Cuevas Martínez, A. A. (2017). Usando TIC para enseñar Matemática en preescolar: El Circo Matemático. Revista Cubana de Ciencias Informáticas, 11(1), 168-181.

Linares, S. (2018). La formación del docente de matemáticas. Realidades y desafíos. Cuadernos de Investigación y Formación en Educación Matemática, 55-61.

Lubert, C. D., Ruiz-Ortega, F. J., \& Londoño, D. M. M. (2021). Conciencia metacognitiva en docentes de diferentes áreas de dominio en una universidad pública de Manizales (Colombia). Tecné, Episteme y Didaxis: TED, (50).

Luna, A. R. A., \& Páez, D. A. (2018). Conocimiento del profesor universitario para enseñar matemáticas. Énfasis en lo pedagógico. DOCERE, (19), 13-16. 
Matamorros Espinoza, W. (2018). Propuesta Didáctica de Aprendizaje basado en Problemas dirigida al Área de Matemáticas $\left(8^{\circ}\right.$ de Educación General Básica): Caso Unidad Educativa "Sagrada Familia". [Trabajo de Titulación previo a la Obtención del Título de Master en Ciencias de la Educación Pontificia Universidad Católica del Ecuador]. Repositorio de la Pontificia Universidad Católica del Ecuador.

Maher, C. A., Sigley, R., Sullivan, P., \& Wilkinson, L. C. (2018). An international perspective on knowledge in teaching mathematics. The Journal of Mathematical Behavior, 51, 71-79.

Mato-Vázquez, D., Chao-Fernández, R., \& Chao-Fernández, A. (2019). Efectos de enseñar matemáticas a través de actividades musicales. Revista latinoamericana de investigación en matemática educativa, 22(2), 163-184.

Muhtadi, D., Kartasasmita, B. G., \& Prahmana, R. C. I. (2017). The Integration of technology in teaching mathematics. In Journal of Physics: Conference Series, 943(1), 012020.

Neyra Quezada, E. R. (2020). Aprendizaje basado en problemas para el aprendizaje significativo en matemática, en estudiantes de tercer año de secundaria, Chao 2019

Pachas, C. I. S. (2020). Herramientas tecnológicas en la enseñanza de las matemáticas durante la pandemia COVID-19. HAMUT'AY, 7(2), 46-57.

Pérez-Díaz, H. M. (2021). Enseñar y aprender matemáticas en tiempos de pandemia. Con-Ciencia Boletín Científico de la Escuela Preparatoria No. 3, 8(16), 14-17.
Pérez González, A., Valdés Rojas, M. B., \& Garriga González, A. T. (2019). Estrategia didáctica para enseñar a planificar los procesos de enseñanza y aprendizaje de la matemática. Revista educación, 43(2), 170-188.

Rashidov, A. (2020). Use of differentiation technology in teaching Mathematics. European Journal of Research and Reflection in Educational Sciences Vol, 8(7).

Rodríguez, W. (2020). Investigación sobre la historia del saber profesional de los docentes que enseñan matemáticas: interrogatorios metodológicos. Revista Paradigma, 41, 900-911.

Rodríguez, M. (2020). La alfabetización matemática en profesionales universitarios: imposibilidad o re-significación urgente. Revista Innova Educación, 2(2), 226-244.

Rodríguez Tortosa, M. (2018). ABP, una propuesta para la clase de matemáticas. [Máster en Profesorado de Educación Secundaria Obligatoria y Bachillerato, Formación Profesional y Enseñanza de Idiomas.Universidad de Almería]. Repositorio Institucional de la Universidad de Almería.

Sánchez-Luján, B. I. (2017). Aprender y enseñar matemáticas: desafío de la educación. IE Revista de Investigación Educativa de la REDIECH, 8(15), 7-10.

Simamora, R. E., Sidabutar, D. R., \& Surya, E. (2017). Improving learning activity and students' problem-solving skill through problem-based learning (PBL) in junior high school. International Journal of Sciences: Basic and Applied Research (IJSBAR), 33(2), 321-331. 
Soledad, L. P. C. (2020). Estrategia ABP para desarrollar competencias en la asignatura matemática básica en la Universidad Peruana Los Andes. La Merced-2017.

Soboleva, E. V., Sabirova, E. G., Babieva, N. S., Sergeeva, M. G., \& Torkunova, J. V. (2021). Formation of Computational Thinking Skills Using Computer Games in Teaching Mathematics. Eurasia Journal of Mathematics, Science and Technology Education, 17(10), em2012.

Thurm, D., \& Barzel, B. (2020). Effects of a professional development program for teaching mathematics with technology on teachers' beliefs, self-efficacy and practices. ZDM, 52(7), 1411-1422.

Vargas, N. (2019). Aprendizaje Basado en Proyectos mediados por TIC para superar dificultades en el aprendizaje de operaciones básicas matemáticas. [Maestría en TIC aplicado a las Ciencias de la Educación. Universidad Pedagógica y Tecnológica de Colombia]. Repositorio Institucional de la Universidad Pedagógica y Tecnológica de Colombia.

Vargas-Pulido, D. A. (2021). Aprendizaje Basado en Problemas y su Influencia en el Aprendizaje de Sistema de Ecuaciones Lineales 2x2 en los Estudiantes del 9-4 de la Institución Educativa Nuestra Señora de los Dolores de Manare de Villanueva Casanare 2019-Colombia.

Vélez, J. J. T., Vizcaíno, C. F. G., Álvarez, J. C. E., \& Zurita, I. N. (2020). Aprendizaje Basado en Problemas como estrategia didáctica para el desarrollo del razonamiento lógico matemático. Revista Arbitrada Interdisciplinaria Koinonía, 5(1), 753-772. 